\title{
Encuesta nacional de salud 2009-2010: Enseñanzas y desafíos
}

\author{
Chilean national health survey 2009-2010: Lessons and challenges
}

De la misma forma que para los médicos es necesario recurrir al uso de métodos, instrumentos y pruebas para establecer la condición de enfermo de una persona, el diagnóstico de la situación de salud de una comunidad completa se establece mediante el uso de instrumentos similares representados por las encuestas poblacionales (Surveys).

A partir del año 2000, nuestro país tomó la decisión de aplicar encuestas de salud para poder cuantificar y vigilar la situación de salud de la población chilena. Mediante un proceso sugerido técnicamente por organismos internacionales ${ }^{1}$, Chile ha sometido progresivamente, a su población a estudios poblacionales. Inicialmente se introdujo la aplicación de cuestionarios de autorreporte (Encuestas de Calidad de Vida 2000 y 2006), y posteriormente se agregaron cuestionarios especificos y mediciones biofisiológicas y bioquímicas de complejidad creciente dando origen a nuestra primera encuesta nacional de salud (ENS 2003), aplicada a una muestra de personas de 17 años y más en todo el país.

Las encuestas poblacionales regulares constituyen hoy en día elementos indispensables para orientar las políticas públicas en salud, puesto que apuntan a los problemas medulares que afligen a la población, cuantificándolos y caracterizándolos para el diseño y aplicación de intervenciones adecuadas.

La "instantánea" obtenida el año 2003, reveló un país con importantes problemas de salud de orden crónicos, con cifras preocupantes de presión arterial elevada, de diabetes, dislipidemia y riesgo cardiovascular. Resultaron también importantes los problemas nutricionales traducidos en altas cifras de sobrepeso y obesidad. Por el lado de las conductas y hábitos, Chile mostró una cara sedentaria, con severo deterioro de salud oral, salud mental y proclive al elevado consumo de tabaco en su población ${ }^{2}$.

Los resultados de la ENS2003 permitieron configurar un panorama actualizado de la condición de salud de la población chilena, sobre la base del cual, fue posible operacionalizar inactivas relevantes concretadas en algunas de las patologías GES del sistema de garantías explícitas en salud (AUGE).

Algunas iniciativas legales relevantes fueron concretadas posterior a la ENS2003, siendo una de las más importantes para nuestra Sociedad Cientifica, la modificación de la ley de tabaco que regula la venta y publicidad y restringe su consumo.

Con la finalidad de actualizar este diagnóstico, incrementar el número de problemas de salud a evaluar y efectuar seguimiento de algunas enfermedades, se llevó a cabo la segunda encuesta nacional de salud 2009-2010, ejecutada al igual que la primera por la Pontificia Universidad Católica de Chile.

Esta segunda encuesta (ENS 2009-10), que tiene representación nacional, regional y según sexo y edad, consideró a algo más de 5.000 chilenos seleccionados aleatoriamente. Se incluyeron en esta versión algunos problemas de salud previamente evaluados (tabaquismo, hipertensión arterial, diabetes, sedentarismo, riesgo cardiovascular, salud mental, estado nutricional), incorporando y mejorando nuevos módulos (consumo de alcohol, actividad fisica, módulo de cobertura preventiva en cáncer, prevención en cáncer, consumo de sal, exposición involuntaria a humo de tabaco y trastornos del sueño) ${ }^{3}$.

Los resultados obtenidos muestran un panorama nacional preocupante, constatándose la persistencia de un elevado nivel de morbilidad por enfermedades crónicas y de sus factores de riesgo. Entre las cifras más preocupantes destacan la presión arterial elevada (26,9\%), dislipidemia HDL $(22,7 \%)$, sobrepeso y obesidad (64\%), diabetes $(9,4 \%)$ y probable consumo elevado de sal (95\%).

En general, los factores de riesgo conductuales evaluados, muestran un perfil preocupante: la población chilena persiste en hábitos alimentarios inadecuados, con escasa actividad física, con alto consumo de alcohol y con deterioro importante de su salud mental. 
La elevada prevalencia de tabaquismo en población adulta (40,6\%) constituye una situación alarmante puesto que en la práctica, la cifra es similar a la encontrada en la EN2003. Adicionalmente, se detectó un incremento en el número de cigarrillos promedio diarios consumidos por los fumadores (10,4 cigarrillos/dia). Es posible hoy contar por primera vez con información poblacional actualizada sobre el nivel de dependencia a la nicotina, el que es de 33,2\% entre los fumadores. Pese a la intensa información disponible sobre los daños del humo de tabaco, en el $30 \%$ de los hogares chilenos encuestados hay aún algún grado de exposición involuntaria al humo de tabaco.

En lo referente a problemas de salud respiratoria, y en cifras similares a la ENS2003, el 24,5\% de la población participante presenta síntomas respiratorios crónicos (Tos, expectoración y algún grado de disnea en forma simultánea). Los diagnósticos autoreportados de Enfermedad Pulmonar obstructiva crónica (5\%) y asma bronquial (6,3\%) son aun infrecuentes en nuestro medio.

Un aporte relevante en el área de enfermedades respiratorias lo constituyó la evaluación de la prevalencia estimada de Síndrome de Apnea Obstructiva del Sueño. Mediante la aplicación de cuestionarios se obtuvo por primera vez una cifra estimada de prevalencia de SAOS en nuestro medio, el que alcanzó una prevalencia de 4,6\% (5,3\% en hombres y 3,9\% en mujeres).

Los hallazgos de esta última encuesta confirman la necesidad de un profundo análisis en el enfrentamiento nacional de la salud de los chilenos. Se requiere adoptar medidas urgentes y posiblemente, efectuar cambios estructurales en las politicas de salud para propiciar, por una parte, instancias para hacer frente a esta carga de morbilidad y por otro, diseñar estrategias de prevención.

Algunas de estas intervenciones requieren de un amplio apoyo social para poder ser concretadas. Entre ellas, sin duda, la mejoría de nuestra actual legislación antitabaco es una prioridad nacional $y$ asi lo ha hecho saber la Sociedad de Enfermedades Respiratorias a las autoridades de salud. En el mismo ámbito, es necesario propiciar una discusión y análisis profundos respecto de la factibilidad de diseñar y poner en acción una estrategia nacional de cesación del hábito tabáquico.

La formulación del actual Plan Nacional de Salud debe recoger la información derivada de la ENS2009-10 y transformarla en estrategias y planes especificos de acuerdo a las prioridades que las autoridades de salud establezcan. En la formulación de dicho Plan, es indispensable la participación de las sociedades cientificas.

Como desafíos futuros para la medicina respiratoria está la oportunidad en próximas versiones de la Encuesta Nacional de Salud, de considerar a nuevos problemas de Salud. Entre ellos, el asma bronquial y la enfermedad pulmonar obstructiva crónica constituyen buenos candidatos para ser evaluados complementariamente, además de las encuestas habituales, con mediciones de función respiratoria y marcadores serológicos, aportando con información indispensable para el mejor conocimiento en nuestro medio de estas enfermedades.

Dr. Gonzalo Valdivia Cabrera

Miembro, Comisión Científica y de Tabaco Sociedad Chilena de Enfermedades Respiratorias

Director ENS 2009-2010

Departamento de Salud Pública,

Pontificia Universidad Católica de Chile.

\section{Bibliografía}

1.- PANAMERICAN STEPS, the PAHO/WHO Stepwise Approach to Chronic noncommunicable Disease RiskFactor Surveillance. http://www.paho.org/english/AD/ DPC/NC/panam-steps.htm. Consultado el 7 de marzo de 2011.

2.- MINISTERIO DE SALUD. Primera Encuesta Nacional de Salud, http://epi.minsal.cl/epi/html/invest/ENS/ENS. htm Consultado el 7 de marzo de 2011.

3.- MINISTERIO DE SALUD. Segunda Encuesta Nacional de Salud, http://www.redsalud.gov.cl/portal/url/item/99 c12b89738d80d5e04001011e0113f8.pdf Consultada el 7 de marzo de 2011. 